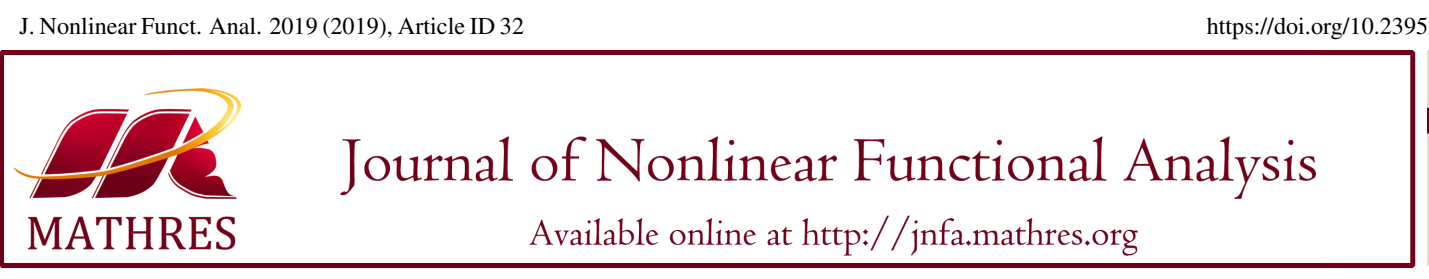

https://doi.org/10.23952/jnfa.2019.32

\title{
A FRACTIONAL $p$-KIRCHHOFF TYPE PROBLEM INVOLVING A PARAMETER
}

\author{
JIABIN ZUO ${ }^{1,2, *}$, TIANQING AN ${ }^{2}$, XIUZHEN LI ${ }^{1}$, YANYING MA ${ }^{1}$ \\ ${ }^{1}$ Faculty of Applied Sciences, Jilin Engineering Normal University, Changchun 130052, China \\ ${ }^{2}$ College of Science, Hohai University, Nanjing 210098, China
}

Abstract. In this paper, by using the symmetric mountain pass theorem and dual fountain theorem, we show the existence of infinitely many solutions for the nonlocal Kirchhoff type equation with the fractional p-Laplacian:

$$
\begin{cases}p \mathscr{M}\left(\iint_{\mathbb{R}^{2 N}} \frac{|u(x)-u(y)|^{p}}{|x-y|^{N+p s}} d x d y\right)(-\triangle)_{p}^{s} u(x)-\lambda|u|^{p-2} u=f(x, u), & \text { in } \Omega \\ u=0, & \text { in } \mathbb{R}^{N} \backslash \Omega\end{cases}
$$

where $\Omega \subset \mathbb{R}^{N}$ is a smooth bounded domain, $(-\triangle)_{p}^{s}$ is a fractional p-Laplace operator with $0<s<1<p<\infty$ and $p s<N$, $\mathscr{M}$ is a continuous function and $\lambda$ is a real parameter.

Keywords. Critical point theory; Dual fountain theorem; Fractional p-Kirchhoff type; Symmetric mountain pass theorem.

2010 Mathematics Subject Classification. 35R11, 35A15.

\section{INTRODUCTION}

Consider the following fractional $p$-Laplacican equations of Kirchhoff type:

$$
\begin{cases}\mathscr{M}\left(\iint_{\mathbb{R}^{2 N}}|u(x)-u(y)|^{p} K(x-y) d x d y\right) \mathscr{L}_{K}^{p} u-\lambda|u|^{p-2} u=f(x, u), & \text { in } \Omega, \\ u=0, & \text { in } \mathbb{R}^{N} \backslash \Omega,\end{cases}
$$

where $N>p s$ with $0<s<1, \Omega$ is a bounded domain with Lipschitz boundary $\partial \Omega, \mathscr{M} \in C\left(\mathbb{R}_{0}^{+}, \mathbb{R}^{+}\right)$ and $\mathscr{L}_{K}^{p}$ is a non-local operator defined by:

$$
\mathscr{L}_{K}^{p} u(x)=2 \lim _{\varepsilon \searrow 0} \int_{\mathbb{R}^{N} \backslash B_{\varepsilon}(x)}|u(x)-u(y)|^{p-2}(u(x)-u(y)) K(x-y) d y \quad x \in \mathbb{R}^{N},
$$

${ }^{*}$ Corresponding author.

E-mail addresses: zuojiabin88@163.com (J. Zuo), antq@hhu.edu.cn (T. An), 540860393@qq.com (X. Li), 710819023 @qq.com (Y. Ma).

Received April 17, 2019; Accepted July 14, 2019.

(C)2019 Journal of Nonlinear Functional Analysis 
where $1<p<\infty, K: \mathbb{R}^{N} \backslash\{0\} \rightarrow(0, \infty)$ is a measurable functional and satisfies the following properties:

$$
\left\{\begin{array}{l}
\gamma k \in L^{1}\left(\mathbb{R}^{N}\right), \text { where } \gamma(\mathrm{x})=\min \left\{|\mathrm{x}|^{\mathrm{p}}, 1\right\} \\
\text { there exists } \mathrm{k}_{0}>0 \text { such that } \mathrm{K}(\mathrm{x}) \geq \mathrm{k}_{0}|\mathrm{x}|^{-(\mathrm{N}+\mathrm{ps})} \text { for any } \mathrm{x} \in \mathbb{R}^{\mathrm{N}} \backslash\{0\} \\
K(-x)=K(x) \text { for any } \mathrm{x} \in \mathbb{R}^{\mathrm{N}} \backslash\{0\}
\end{array}\right.
$$

If $K$ is a standard type, i.e., $K(x)=|x|^{-(N+p s)}$, then problem (1.1) becomes

$$
\begin{cases}\mathscr{M}\left(\iint_{\mathbb{R}^{2 N}} \frac{|u(x)-u(y)|^{p}}{|x-y|^{N+p s}} d x d y\right)(-\triangle)_{p}^{s} u(x)-\lambda|u|^{p-2} u=f(x, u), & \text { in } \Omega, \\ u=0, & \text { in } \mathbb{R}^{\mathrm{N}} \backslash \Omega,\end{cases}
$$

where $(-\triangle)_{p}^{s}$ is a fractional p-Laplace operator defined by

$$
(-\triangle)_{p}^{s} u(x)=2 \lim _{\varepsilon \rightarrow 0^{+}} \int_{\mathbb{R}^{N} \backslash B_{\varepsilon}(x)} \frac{|u(x)-u(y)|^{p-2}(u(x)-u(y))}{|x-y|^{N+p s}} d y
$$

for $x \in \mathbb{R}^{N}$.

Clearly, if $p=2, \mathscr{M}=1, \lambda=0$, then equation (1.3) is reduced to the following form:

$$
\begin{cases}(-\triangle)^{s} u=f(x, u), & \text { in } \Omega, \\ u=0, & \text { in } \mathbb{R}^{\mathrm{N}} \backslash \Omega .\end{cases}
$$

Recently, a lot of results were obtained for above problems, see, for example, $[1,2,3,4,5,6,7,8,9,10$, $11,12,13,14,15]$ and references therein. Especially, some important existence results for infinite many solutions were proved in $[16,17,18]$.

The purpose of this paper is to discuss the existence of infinite many solutions of problem (1.1). We make the following assumptions on functions $\mathscr{M}$ and $f$.

$\left(M_{1}\right) \mathscr{M} \in C\left(\mathbb{R}_{0}^{+}, \mathbb{R}^{+}\right)$satisfies $\inf _{t \in \mathbb{R}_{0}^{+}} \mathscr{M}(t) \geq m_{0}>0$, where $m_{0}$ is a positive constant.

$\left(M_{2}\right)$ There exists $\theta \in\left[1, \frac{N}{N-p s}\right)$ such that

$$
\theta \widetilde{\mathscr{M}}(t)=\theta \int_{0}^{t} \mathscr{M}(s) d s \geq \mathscr{M}(t) t, \forall t \in \mathbb{R}_{0}^{+} .
$$

$\left(f_{1}\right)$ There is a positive constant $C>0$ such that

$$
|f(x, t)| \leq C\left(|t|^{q-1}+1\right)
$$

for some $q \in\left(p, p_{s}^{*}\right)$ and every $x \in \Omega, t \in \mathbb{R}$, where $p_{s}^{*}=\frac{p N}{N-p s}$.

$\left(f_{2}\right)$ There exists $\mu>p \theta$ such that

$$
F(x, t)=\int_{0}^{t} f(x, s) d s \leq \frac{1}{\mu} f(x, t) t \text { for any } \mathrm{x} \in \Omega, \mathrm{t} \in \mathbb{R} .
$$

$\left(f_{3}\right) \lim _{|t| \rightarrow \infty} \frac{F(x, t)}{|t|^{\theta \theta}} \rightarrow+\infty$ uniformly for a.e. $x \in \Omega$.

$\left(f_{4}\right) f(x, t)$ is odd for $t$, i.e. $-f(x,-t)=f(x, t)$ for each $x \in \Omega$ and $t \in \mathbb{R}^{N}$.

$\left(f_{5}\right) \lim _{|t| \rightarrow 0} \frac{f(x, t)}{|t|^{p-1}}=0$ uniformly for $x \in \Omega$.

Since we assume here that $\mu>p \theta$, we know that condition $\left(f_{2}\right)$ is different from the usual (AR) condition. 
Definition 1.1. We say that $u \in X_{0}$ is a weak solution of problem (1.1) if

$$
\begin{gathered}
\mathscr{M}\left(\|u(x)\|_{X_{0}}^{p}\right) \iint_{\mathbb{R}^{2 N}}|u(x)-u(y)|^{p-2}(u(x)-u(y))(\varphi(x)-\varphi(y) K(x-y) d x d y \\
-\lambda \int_{\Omega}|u(x)|^{p-2} u(x) \varphi(x) d x-\int_{\Omega} f(x, u(x)) \varphi(x) d x=0
\end{gathered}
$$

for any $\varphi \in X_{0}$. This space $X_{0}$ will be introduced in the second part.

The main results of this paper are as follows.

Theorem 1.2. Let $K: \mathbb{R}^{N} \backslash 0 \rightarrow(0, \infty)$ be a function fulfilling (1.2). If $\left(M_{1}\right)-\left(M_{2}\right)$ and $\left(f_{1}\right)-\left(f_{5}\right)$ hold, then problem (1.1) has infinitely many nontrivial solutions $\left\{u_{k}\right\}$ in $X_{0}$ with unbounded energy for every $\lambda \in \mathbb{R}$.

Theorem 1.3. Let $K: \mathbb{R}^{N} \backslash 0 \rightarrow(0, \infty)$ be a function fulfilling (1.2). If $\left(M_{1}\right)-\left(M_{2}\right)$ hold and $f(x, t)=$ $\alpha|t|^{\xi-2} t+\beta|t|^{\eta-2} t$ with $1<\xi<p<p \theta<\eta \leq q<p_{s}^{*}$, where $\theta$ is given in $\left(M_{2}\right)$, then there is a constant $\Lambda>0$ such that, for any $\lambda \leq \Lambda, \alpha>0, \beta \in \mathbb{R}$, problem (1.1) has a sequence nontrivial negative energy solutions $\left\{u_{k}\right\}$ in $X_{0}$ with converging to 0 .

Our results improve some existing results in three folds. Problem (1.1) involving parameters is studied. The dual fountain theorem was used only when $p=2$ in [16], and the nonlinear condition is also stronger than ours. In [18], Nyamoradi and Zaidan only considered the situation that $\mathscr{M}=a+b t$ without the parameters. The proof of Theorem 1.2 is different from [16]. Our results are more general. The framework of this paper is as follows. In Section 2, we introduce necessary preliminaries. In Section 3, we verify the Cerami condition for our functional. In last section, Section 4, our main results are presented.

\section{VARIATIONAL FRAMEWORK}

In this section, we first review some basic variational frameworks and some useful Lemmas that will be used in the next section for problem (1.1). Let $Q=\mathbb{R}^{2 N} \backslash \mathscr{O}$, where

$$
\mathscr{O}=\mathscr{C}(\Omega) \times \mathscr{C}(\Omega) \subset \mathbb{R}^{2 N}
$$

and $\mathscr{C}(\Omega)=\mathbb{R}^{N} \backslash \Omega$. $X$ is a Lebesgue measurable functions linear space. Such that any function $u \in X$ limited to $\Omega$ belongs to $L^{p}(\Omega)$ and

$$
\int_{Q}|u(x)-u(y)|^{p} K(x-y) d x d y<\infty
$$

The space $\mathrm{X}$ is given by a norm:

$$
\|u\|_{X}=\|u\|_{L^{p}(\Omega)}+\left(\int_{Q}|u(x)-u(y)|^{p} K(x-y) d x d y\right)^{\frac{1}{p}} .
$$

It is readily seen that $\|\cdot\|_{X}$ is a norm on $X$ and $C_{0}^{\infty}(\Omega) \subset X$ (see [7, Lemma 2.1]). The space in which we work is a closed linear subspace $X_{0} \subset X$,

$$
X_{0}=\left\{u \in X: u(x)=0 \text { a.e. in } \mathbb{R}^{\mathrm{N}} \backslash \Omega\right\} .
$$

Also, we note that $X_{0}$ can be endowed with the norm

$$
\|u\|_{X_{0}}=\left(\int_{Q}|u(x)-u(y)|^{p} K(x-y) d x d y\right)^{\frac{1}{p}} \text { for all } \mathrm{u} \in \mathrm{X}_{0}
$$


and $\left(X_{0},\|\cdot\|_{X_{0}}\right)$ is a uniformly convex reflexive Banach space (see [17, Remark 2.1 and Lemma 2.4]).

Lemma 2.1. [7] Let $K: \mathbb{R}^{N} \backslash\{0\} \rightarrow(0, \infty)$ be a function which fulfills (1.2) and let $\left\{u_{j}\right\}$ be a bounded sequence in $X_{0}$. Then exists $u \in L^{v}\left(\mathbb{R}^{N}\right)$ with $u=0$ a.e. in $\mathbb{R}^{N} \backslash \Omega$ such that up to a subsequence,

$$
u_{j} \rightarrow u \text { strongly in } L^{v}(\Omega), \text { as } \mathrm{j} \rightarrow \infty
$$

for any $v \in\left[1, p_{s}^{*}\right)$.

Remark 2.2. $X_{0} \hookrightarrow \hookrightarrow L^{v}(\Omega)$, for each $v \in\left[1, p_{s}^{*}\right)$, Moreover, there exists $C_{v}>0$ such that

$$
\|u\|_{v} \leq C_{v}\|u\|_{X_{0}}, u \in X_{0}
$$

We need to review some properties of the eigenvalue problem and the spectrum of the operator. For more details, the reader is referred to [19] and the references therein.

$$
\begin{cases}\mathscr{L}_{K}^{p} u=\lambda_{k}|u|^{p-2} u, & \text { in } \Omega, \\ u=0, & \text { in } \mathbb{R}^{\mathrm{N}} \backslash \Omega,\end{cases}
$$

there is a divergent positive eigenvalue sequence

$$
\lambda_{1}<\lambda_{2} \leq \cdots \leq \lambda_{k} \leq \lambda_{k+1} \leq \cdots,
$$

whose eigenvalues are the critical values of the functional

$$
I_{p}(u)=\|u\|_{X_{0}}^{p}, u \in \Sigma=\left\{u \in X_{0}: \int_{\Omega}|u|^{p} d x=1\right\} .
$$

We notice that the first eigenvalue $\lambda_{1}:=\inf _{u \in \Sigma} I_{p}(u)>0$. The corresponding eigenfunctions will be denoted by $e_{j}$. Let $X_{j}=\operatorname{span}\left\{e_{j}\right\}$. Then

$$
X_{0}=\overline{\bigoplus_{i=1}^{\infty} X_{i}}, Y_{k}=\bigoplus_{i=1}^{k} X_{i}, Z_{k}=\overline{\bigoplus_{i=k}^{\infty} X_{i}}, k=1,2, \cdots
$$

Let $B_{k}:=\left\{u \in Y_{k}:\|u\|_{X_{0}} \leq r_{k}\right\}, N_{k}:=\left\{u \in Z_{k}:\|u\|_{X_{0}}=\gamma_{k}\right\}$, where $r_{k}>\gamma_{k}>0$.

We need the following definition, which is a weak version of the (PS).

Definition 2.3. Let $I \in C^{1}(X, \mathbb{R})$. We say that I satisfies the $(C e)_{c}$ condition at the level $c \in \mathbb{R}$ if any sequence $\left\{u_{n}\right\}_{n} \subset X_{0}$ such that

$$
I\left(u_{n}\right) \rightarrow c,\left(1+\left\|u_{n}\right\|\right) I^{\prime}\left(u_{n}\right) \rightarrow 0 \text { in } X_{0}^{\prime} \text { as } n \rightarrow \infty,
$$

where $X_{0}^{\prime}$ is the dual space of $X_{0}$, possesses a convergent subsequence in $X_{0}$; I satisfies the (Ce) condition if I satisfies the $(\mathrm{Ce})_{c}$ for all $c \in \mathbb{R}$.

Definition 2.4. Let $I \in C^{1}(X, \mathbb{R})$. We say that I satisfies the $(C e)_{c}^{*}$ condition at the level $c \in \mathbb{R}$ (with respect to $\left.Y_{n}\right)$ if any sequence $\left\{u_{n_{j}}\right\}_{n_{j}} \subset X_{0}$ such that

$$
u_{n_{j}} \in Y_{n_{j}}, I\left(u_{n_{j}}\right) \rightarrow c,\left(1+\left\|u_{n_{j}}\right\|\right) I_{Y_{n_{j}}}^{\prime}\left(u_{n_{j}}\right) \rightarrow 0 \text { in } X_{0}^{\prime} \text { as } n_{j} \rightarrow \infty
$$

possesses a convergent subsequence in $X_{0}$; I satisfies the $(\mathrm{Ce})^{*}$ condition if I satisfies the $(\mathrm{Ce})_{c}^{*}$ for all $c \in \mathbb{R}$. 
Theorem 2.5. (Symmetric Mountain Pass Theorem, [18]) Let $X_{0}$ be a real infinite dimensional Banach space and $X_{0}=Y \bigoplus Z$, where $Y$ is a finite subspace. If $I \in C^{1}\left(X_{0}, \mathbb{R}\right)$ satisfies $(C e)_{c}$ conditional for every $\mathrm{c}$ and the following three conditions:

$\left(A_{1}\right) I(0)=0, I(-u)=I(u)$ for all $u \in X_{0}$;

$\left(A_{2}\right)$ there exist constants $\alpha, \rho>0$ such that $\left.I\right|_{\partial B_{\rho} \cap Y} \geq \alpha$, where

$$
B_{\rho}=\left\{u \in X_{0}:\|u\|_{X_{0}} \leq \rho\right\}
$$

$\left(A_{3}\right)$ for any finite dimensional subspace $\widetilde{X} \subset X_{0}$, there exists $R=R(\widetilde{X})>0$ such that $I \leq 0$ as $u \in \widetilde{X} \backslash B_{\rho}$. Then I possesses an unbounded sequence of critical values.

Theorem 2.6. (Dual Fountain Theorem, [16]) Assume that $I \in C^{1}\left(X_{0}, \mathbb{R}\right)$ satisfies $I(-u)=I(u)$. Suppose that, for every $k \geq k_{0}$, there exists $r_{k}>\gamma_{k}>0$ such that

$\left(B_{1}\right) a_{k}=\inf \left\{I(u): u \in Z_{k},\|u\|_{X_{0}}=r_{k}\right\} \geq 0$

$\left(B_{2}\right) b_{k}=\max \left\{I(u): u \in Y_{k}:\|u\|_{X_{0}}=\gamma_{k}\right\} \leq 0$;

$\left(B_{3}\right) d_{k}=\inf \left\{I(u): u \in Z_{k}:\|u\|_{X_{0}} \leq \gamma_{k}\right\} \rightarrow 0$ as $k \rightarrow \infty$;

$\left(B_{4}\right) I$ satisfies the $(C e)_{c}^{*}$ condition for every $c \in\left[d_{k_{0}}, 0\right]$.

Then I has a sequence of negative critical values converging to 0 .

\section{COMPaCtness CONDitions}

For $u \in X_{0}$, we define energy functional $I: X_{0} \rightarrow \mathbb{R}$ associated with problem (1.1). Set

$$
I(u)=T(u)-J(u)-H(u),
$$

where

$$
T(u)=\frac{1}{p} \widetilde{\mathscr{M}}\left(\|u\|_{X_{0}}^{p}\right)
$$

and

$$
J(u)=\frac{\lambda}{p} \int_{\Omega}|u|^{p} d x, H(u)=\int_{\Omega} F(x, u) d x .
$$

Lemma 3.1. [7] If $\left(M_{1}\right)$ holds, then $T: X_{0} \rightarrow \mathbb{R}$ is of class $C^{1}\left(X_{0}, \mathbb{R}\right)$, and

$$
\left\langle T^{\prime}(u), v\right\rangle=\mathscr{M}\left(\|u(x)\|_{X_{0}}^{p}\right) \iint_{\mathbb{R}^{2 N}}|u(x)-u(y)|^{p-2}(u(x)-u(y))(v(x)-v(y) K(x-y) d x d y
$$

for all $u, v \in X_{0}$. Moreover, for each $u \in X_{0}, T^{\prime}(u) \in X_{0}^{\prime}$.

Lemma 3.2. [7] Let $\left(f_{1}\right)$ holds, then the functional $H$ is of $\operatorname{class} C^{1}\left(X_{0}, \mathbb{R}\right)$, and

$$
\left\langle H^{\prime}(u), v\right\rangle=\int_{\Omega} f(x, u) v d x
$$

for all $u, v \in X_{0}$.

Combining Lemma 3.1 with Lemma 3.2, we get that $I \in C^{1}\left(X_{0}, \mathbb{R}\right)$ and

$$
\begin{gathered}
\left\langle I^{\prime}(u), v\right\rangle=\mathscr{M}\left(\|u(x)\|_{X_{0}}^{p}\right) \\
\iint_{\mathbb{R}^{2 N}}|u(x)-u(y)|^{p-2}(u(x)-u(y))(v(x)-v(y) K(x-y) d x d y \\
-\lambda \int_{\Omega}|u|^{p-2} u v d x-\int_{\Omega} f(x, u) v d x
\end{gathered}
$$

for all $u, v \in X_{0}$.

Clearly, weak solutions of problem (1.1) are the critical points of energy functional $I$. 
Lemma 3.3. Assume that $\left(M_{1}\right)$ and $\left(f_{1}\right)$ hold. Then any bounded sequence $\left\{u_{n}\right\}_{n} \subset X_{0}$ with $(1+$ $\left.\left\|u_{n}\right\|\right) I^{\prime}\left(u_{n}\right) \rightarrow 0$ as $n \rightarrow \infty$ has a strongly convergent subsequence in $X_{0}$.

Proof. Suppose that $\left\{u_{n}\right\}_{n} \subset X_{0}$ is bounded sequence. By Lemma 2.1, due to the reflexivity of $X_{0}$, there is a subsequence which is still denoted by $\left\{u_{n}\right\}_{n}$ such that

$$
\begin{aligned}
& u_{n} \rightarrow u \text { in } \mathrm{X}_{0}, \\
& u_{n} \rightarrow u \text { in } \mathrm{L}^{\mathrm{v}}(\Omega), 1 \leq \mathrm{v}<\mathrm{p}_{\mathrm{s}}^{*}, \\
& u_{n} \rightarrow u \text { a.e. in } \mathbb{R}^{\mathrm{N}} .
\end{aligned}
$$

To prove $\left\{u_{n}\right\}_{n} \rightarrow u$, in $X_{0}$, we first introduce some simple notations. Let $\varphi \in X_{0}$ be fixed and let $B_{\varphi}$ be the linear functional on $X_{0}$ defined by

$$
B_{\varphi}(v)=\iint_{\mathbb{R}^{2 N}}|\varphi(x)-\varphi(y)|^{p-2}(\varphi(x)-\varphi(y))(v(x)-v(y) K(x-y) d x d y
$$

for all $v \in X_{0}$.

Obviously, by the Hölder inequality and the continuity of $B_{\varphi}$, we have

$$
\left|B_{\varphi}(v)\right| \leq\|\varphi\|_{X_{0}}^{p-1}\|v\|_{X_{0}} \text { for all } \mathrm{v} \in \mathrm{X}_{0} .
$$

From the weak convergence of $\left\{u_{n}\right\}$ in $X_{0}$, we get

$$
\lim _{n \rightarrow \infty} B_{u}\left(u_{n}-u\right)=0 \text {. }
$$

It is easy to see $\left\langle I^{\prime}\left(u_{n}\right), u_{n}-u\right\rangle \rightarrow 0$, if $u_{n} \rightarrow u$ in $X_{0}$, and $\left(1+\left\|u_{n}\right\|\right) I^{\prime}\left(u_{n}\right) \rightarrow 0$. So,

$$
\begin{aligned}
\left\langle I^{\prime}\left(u_{n}\right), u_{n}-u\right\rangle= & \mathscr{M}\left(\left\|u_{n}(x)\right\|_{X_{0}}^{p}\right) B_{u_{n}}\left(u_{n}-u\right)-\lambda \int_{\Omega}\left|u_{n}\right|^{p-2} u_{n}\left(u_{n}-u\right) d x \\
& -\int_{\Omega} f\left(x, u_{n}\right)\left(u_{n}-u\right) d x \rightarrow 0 \text { as } \mathrm{n} \rightarrow \infty .
\end{aligned}
$$

Furthermore, according to Lemma 2.1, we know that there is a subsequence,

$$
u_{n} \rightarrow u \text { strongly in } \mathrm{L}^{\mathrm{v}}(\Omega) \text { and a.e. in } \Omega \text {. }
$$

So, $f\left(x, u_{n}\right)\left(u_{n}-u\right) \rightarrow 0$ a.e. in $\Omega$ as $n \rightarrow \infty$. It is clear that sequence $\left\{f\left(x, u_{n}\right)\left(u_{n}-u\right)\right\}$ is not only uniformly bounded but also equi-integrable in $L^{1}(\Omega)$. By the Vitali Convergence Theorem (see [20]), we get

$$
\lim _{n \rightarrow \infty} \int_{\Omega} f\left(x, u_{n}\right)\left(u_{n}-u\right) d x=0 .
$$

Since the second term converges to 0 , we have from (3.2) that

$$
\mathscr{M}\left(\left\|u_{n}(x)\right\|_{X_{0}}^{p}\right) B_{u_{n}}\left(u_{n}-u\right) \rightarrow 0, \text { as } \mathrm{n} \rightarrow 0 .
$$

It follows from $\left(M_{1}\right)$ that $\mathscr{M}(t)$ is bounded on closed interval. Hence

$$
B_{u_{n}}\left(u_{n}-u\right) \rightarrow 0 \text { as } \mathrm{n} \rightarrow \infty
$$

Combining (3.1) with (3.3), we get

$$
\left(B_{u_{n}}\left(u_{n}-u\right)-B_{u}\left(u_{n}-u\right)\right) \rightarrow 0 \text { as } \mathrm{n} \rightarrow 0 .
$$

Using the Simon inequalities:

$$
\left(|\zeta|^{p-2} \zeta-|\eta|^{p-2} \eta\right) \cdot(\zeta-\eta) \geq C_{P}|\zeta-\eta|^{p}, p \geq 2
$$




$$
\left(|\zeta|^{p-2} \zeta-|\eta|^{p-2} \eta\right) \cdot(\zeta-\eta) \geq \widehat{C_{P}} \frac{|\zeta-\eta|^{2}}{(|\zeta|+|\eta|)^{2-p}}, 1<p<2
$$

for all $\zeta, \eta \in \mathbb{R}^{N}$, where $C_{P}, \widehat{C_{P}}>0$ depend only on $p$. Therefore, it follows from (3.4) that we first assume $p \geq 2$. Then, as $n \rightarrow \infty$, we have

$$
\begin{aligned}
\left\|u_{n}-u\right\|_{X_{0}}^{p} & \leq \frac{1}{C_{P}} \iint_{\mathbb{R}^{2 N}}\left(\left|u_{n}(x)-u_{n}(y)\right|^{p-2}\left(u_{n}(x)-u_{n}(y)\right)-|u(x)-u(y)|^{p-2}(u(x)-u(y))\right) \\
& \times\left(u_{n}(x)-u(x)-u_{n}(y)+u(y)\right) K(x-y) d x d y \\
& =\frac{1}{C_{P}}\left(B_{u_{n}}\left(u_{n}-u\right)-B_{u}\left(u_{n}-u\right)\right)=o(1) .
\end{aligned}
$$

So, $\left\|u_{n}-u\right\|_{X_{0}}^{p} \rightarrow 0$ as $n \rightarrow \infty$.

Finally, it remains to take into account the situation when $1<p<2$. From the Hölder inequality, the Simon inequality, and (3.4), we know that, as $n \rightarrow \infty$,

$$
\begin{aligned}
\left\|u_{n}-u\right\|_{X_{0}}^{p} & \leq \frac{1}{\widehat{C}_{P}^{\frac{p}{2}}}\left(B_{u_{n}}\left(u_{n}-u\right)-B_{u}\left(u_{n}-u\right)\right)^{\frac{p}{2}}\left(\left\|u_{n}\right\|_{X_{0}}^{p}+\|u\|_{X_{0}}^{p}\right)^{\frac{2-p}{2}} \\
& \leq \frac{1}{\widehat{C}_{P}^{\frac{p}{2}}}\left(B_{u_{n}}\left(u_{n}-u\right)-B_{u}\left(u_{n}-u\right)\right)^{\frac{p}{2}}\left(\left\|u_{n}\right\|_{X_{0}}^{p(2-p) / 2}+\|u\|_{X_{0}}^{p(2-p) / 2}\right) \\
& =C\left(B_{u_{n}}\left(u_{n}-u\right)-B_{u}\left(u_{n}-u\right)\right)^{\frac{p}{2}}=o(1)
\end{aligned}
$$

where $C>0$. Combing the above two cases, we know that $u_{n} \rightarrow u$ in $X_{0}$ as $n \rightarrow \infty$.

Theorem 3.4. Under the conditions of Theorem 1.2, functional I fulfills the $(\mathrm{Ce})_{c}$ condition.

Proof. By Lemma 3.3, it is sufficient to show the boundedness of $(\mathrm{Ce})_{c}$ sequences. Let $\left\{u_{n}\right\}_{n} \subset X_{0}$ be a $(\mathrm{Ce})_{c}$ sequences for $c \in \mathbb{R}$ such that

$$
I\left(u_{n}\right) \rightarrow c,\left(1+\left\|u_{n}\right\|\right) I^{\prime}\left(u_{n}\right) \rightarrow 0 \text { as } \mathrm{n} \rightarrow \infty .
$$

We first claim that $\left\{u_{n}\right\}$ is a bounded sequence. Assume to the contrary that $\left\|u_{n}\right\|_{X_{0}} \rightarrow \infty$. We consider $\varpi_{n}:=\frac{u_{n}}{\left\|u_{n}\right\|_{X_{0}}}$. Then $\left\|\varpi_{n}\right\|_{X_{0}}=1$. Without loss of generality, we may suppose that

$$
\begin{aligned}
& \varpi_{n} \rightarrow \varpi \text { in } \mathrm{X}_{0} ; \\
& \varpi_{n} \rightarrow \varpi \text { in } \mathrm{L}^{\mathrm{v}}(\Omega), 1 \leq \mathrm{v}<\mathrm{p}_{\mathrm{s}}^{*} ; \\
& \varpi_{n} \rightarrow \varpi \text { a.e. in } \mathbb{R}^{\mathrm{N}}
\end{aligned}
$$

as $n \rightarrow \infty$. There are only two situations which need to be taken into account: $\Phi=0$ or $\Phi \neq 0$. We first consider the case $\varpi=0$. By $\left(f_{2}\right),\left(M_{2}\right)$ and (3.5), we obtain 


$$
\begin{aligned}
\frac{1}{\left\|u_{n}\right\|_{X_{0}}^{p}}\left(I\left(u_{n}\right)\right. & \left.-\frac{1}{\mu} I^{\prime}\left(u_{n}\right) u_{n}\right) \\
& \geq \frac{1}{\left\|u_{n}\right\|_{X_{0}}^{p}}\left(\frac{1}{p} \widetilde{\mathscr{M}}\left(\left\|u_{n}\right\|_{X_{0}}^{p}\right)-\frac{1}{\mu} \mathscr{M}\left(\left\|u_{n}\right\|_{X_{0}}^{p}\right)\|u\|_{X_{0}}^{p}\right. \\
& \left.+\lambda\left(\frac{1}{\mu}-\frac{1}{p}\right)\left\|u_{n}\right\|_{p}^{p}-\int_{\Omega}\left(F\left(x, u_{n}(x)\right)-\frac{1}{\mu} f\left(x, u_{n}(x)\right) u_{n}(x)\right) d x\right) \\
& \geq \frac{1}{\left\|u_{n}\right\|_{X_{0}}^{p}}\left(\left(\frac{1}{p \theta}-\frac{1}{\mu}\right) \mathscr{M}\left(\left\|u_{n}\right\|_{X_{0}}^{p}\right)\left\|u_{n}\right\|_{X_{0}}^{p}\right)+\lambda\left(\frac{1}{\mu}-\frac{1}{p}\right) \int_{\Omega} \varpi_{n}^{p} d x \\
& \geq m_{0}\left(\frac{1}{p \theta}-\frac{1}{\mu}\right),
\end{aligned}
$$

which implies $0 \geq m_{0}\left(\frac{1}{p \theta}-\frac{1}{\mu}\right)$. This is a contradiction.

For $\varpi \neq 0$, we set $\Omega_{1}:=\{x \in \Omega: \varpi(x) \neq 0\}$. Clearly $\left|\Omega_{1}\right|>0$ where $\left|\Omega_{1}\right|$ is Lebesgue measure of $\Omega_{1}$. For $x \in \Omega_{1}$, we have $\left|u_{n}(x)\right| \rightarrow \infty$ as $n \rightarrow \infty$. In view of $\left(f_{3}\right)$, one has

$$
\lim _{n \rightarrow \infty} \frac{F\left(x, u_{n}(x)\right)}{\left|u_{n}(x)\right|^{p \theta}}\left|\varpi_{n}(x)\right|^{p \theta} \rightarrow+\infty
$$

Consequently, according to Fatou's Lemma, we can get

$$
\lim _{n \rightarrow \infty} \int_{\Omega_{1}} \frac{F\left(x, u_{n}(x)\right)}{\left|u_{n}(x)\right|^{p \theta}}\left|\varpi_{n}(x)\right|^{p \theta} d x \rightarrow+\infty .
$$

From $\left(f_{1}\right)$, we obtain

$$
|F(x, t)| \leq M|t|, \forall x \in \Omega,|t| \leq L_{1},
$$

where $L_{1}$ is a positive constant. Combining the above inequality and $\left(f_{3}\right)$, we get

$$
|F(x, t)| \geq-M|t|, \forall(x, t) \in \Omega \times \mathbb{R},
$$

where $M$ is a positive constant. From (2.1), we obtain

$$
\int_{\Omega \backslash \Omega_{1}} \frac{F\left(x, u_{n}(x)\right)}{\left\|u_{n}(x)\right\|_{X_{0}}^{p \theta}} d x \geq-\frac{M \int_{\Omega \backslash \Omega_{1}}\left|u_{n}\right| d x}{\left\|u_{n}(x)\right\|_{X_{0}}^{p \theta}} \geq-\frac{M\left\|u_{n}\right\|_{1}}{\left\|u_{n}(x)\right\|_{X_{0}}^{p \theta}} \geq-\frac{M C_{1}}{\left\|u_{n}(x)\right\|_{X_{0}}^{p \theta-1}},
$$

which implies

$$
\liminf _{n \rightarrow \infty} \int_{\Omega \backslash \Omega_{1}} \frac{F\left(x, u_{n}(x)\right)}{\left\|u_{n}(x)\right\|_{X_{0}}^{p \theta}} d x \geq 0
$$

where $C_{1}$ is a positive constant. From $\left(M_{2}\right)$, we get

$$
\widetilde{\mathscr{M}}(t) \leq \widetilde{\mathscr{M}}(1) t^{\theta}, \forall t \in[1,+\infty) .
$$


By (3.5), (3.7), (3.8), (3.9), and boundedness of $\left\{\varpi_{n}\right\}$, we have

$$
\begin{aligned}
0 & =\lim _{n \rightarrow \infty} \frac{c+o(1)}{\left\|u_{n}(x)\right\|_{X_{0}}^{p \theta}}=\lim _{n \rightarrow \infty} \frac{I\left(u_{n}\right)}{\left\|u_{n}(x)\right\|_{X_{0}}^{p \theta}} \\
& =\lim _{n \rightarrow \infty} \frac{1}{\left\|u_{n}(x)\right\|_{X_{0}}^{p \theta}}\left(\frac{1}{p} \widetilde{\mathscr{M}}\left(\left\|u_{n}\right\|_{X_{0}}^{p}\right)-\frac{\lambda}{p}\left\|u_{n}\right\|_{p}^{p}-\int_{\Omega} F\left(x, u_{n}\right) d x\right) \\
& \leq \lim _{n \rightarrow \infty} \frac{1}{\left\|u_{n}(x)\right\|_{X_{0}}^{p \theta}}\left(\frac{1}{p} \widetilde{\mathscr{M}}(1)\left\|u_{n}\right\|_{X_{0}}^{p \theta}-\frac{\lambda}{p}\left\|u_{n}\right\|_{p}^{p}-\int_{\Omega_{1}} F\left(x, u_{n}\right) d x-\int_{\Omega \backslash \Omega_{1}} F\left(x, u_{n}\right) d x\right) \\
& \leq \frac{\widetilde{\mathscr{M}}(1)}{p}-\frac{\lambda}{p} \lim _{n \rightarrow \infty} \frac{\left\|\varpi_{n}\right\|_{p}^{p}}{\left\|u_{n}\right\|_{X_{0}}^{p \theta-p}}-\lim _{n \rightarrow \infty} \int_{\Omega_{1}} \frac{F\left(x, u_{n}(x)\right)}{\left\|u_{n}(x)\right\|^{p \theta}} d x-\liminf _{n \rightarrow \infty} \int_{\Omega \backslash \Omega_{1}} \frac{F\left(x, u_{n}(x)\right)}{\left\|u_{n}(x)\right\|_{X_{0}}^{p \theta}} d x \\
& \leq \frac{\widetilde{\mathscr{M}}(1)}{p}-\lim _{n \rightarrow \infty} \int_{\Omega_{1}} \frac{F\left(x, u_{n}(x)\right)}{\left\|u_{n}(x)\right\|^{p \theta}} d x=-\infty,
\end{aligned}
$$

which is a contradiction. Then we claim that $\left\{u_{n}\right\}$ is bounded in $X_{0}$. From Lemma 3.3, we can easily obtain that $\left\{u_{n}\right\}$ has a convergence subsequence. Thus, the functional I satisfies (Ce) condition.

Theorem 3.5. Under the conditions of Theorem 1.3, functional I fulfills the $(\mathrm{Ce})_{c}^{*}$ condition.

Proof. On the basis of Lemma 3.3, we just need to prove that the boundedness of $(\mathrm{Ce})_{c}^{*}$ sequence. Consider a sequence $\left\{u_{n_{j}}\right\}_{n_{j}} \subset X_{0}$ such that

$$
u_{n_{j}} \in Y_{n_{j}}, I\left(u_{n_{j}}\right) \rightarrow c,\left(1+\left\|u_{n_{j}}\right\|\right) I_{Y_{n_{j}}}^{\prime}\left(u_{n_{j}}\right) \rightarrow 0 \text { as } \mathrm{n}_{\mathrm{j}} \rightarrow \infty \text {. }
$$

For $n_{j}$ large enough and from the conditions $\left(M_{1}\right)$ and $\left(M_{2}\right)$, we obtain

$$
\begin{aligned}
1+c & +\left\|u_{n_{j}}\right\|_{X_{0}} \geq I\left(u_{n_{j}}\right)-\frac{1}{\eta}\left\langle I^{\prime}\left(u_{n_{j}}\right), u_{n_{j}}\right\rangle \\
& =\frac{1}{p} \widetilde{\mathscr{M}}\left(\left\|u_{n_{j}}\right\|_{X_{0}}^{p}\right)-\frac{1}{\eta} \mathscr{M}\left(\left\|u_{n_{j}}\right\|_{X_{0}}^{p}\right)\left\|u_{n_{j}}\right\|_{X_{0}}^{p}-\lambda\left(\frac{1}{p}-\frac{1}{\eta}\right)\left\|u_{n_{j}}\right\|_{p}^{p}-\alpha\left(\frac{1}{\xi}-\frac{1}{\eta}\right)\left\|u_{n_{j}}\right\|_{\xi}^{\xi} \\
& \geq\left\{m_{0}\left(\frac{1}{p \theta}-\frac{1}{\eta}\right)-\max \left\{0, \lambda C_{p}^{p}\left(\frac{1}{p}-\frac{1}{\eta}\right)\right\}\right\}\left\|u_{n_{j}}\right\|_{X_{0}}^{p}-\alpha C_{p}^{\xi}\left\|u_{n_{j}}\right\|_{X_{0}}^{\xi} .
\end{aligned}
$$

Pick a number $\Lambda>0$ such that

$$
m_{0}\left(\frac{1}{p \theta}-\frac{1}{\eta}\right)-\lambda C_{p}^{p}\left(\frac{1}{p}-\frac{1}{\eta}\right)>0
$$

for any $\lambda \leq \Lambda$. We can deduce that $\left\{u_{n_{j}}\right\}$ in $X_{0}$ is bounded.

\section{Main Results}

First, we state a lemma which will be used later.

Lemma 4.1. [21, Lemma 6] Let $1 \leq q<p_{s}^{*}$ and, for every $k \in \mathbb{N}$, let

$$
\beta_{k}(q):=\sup \left\{\|u\|_{q}: u \in Z_{k},\|u\|_{X_{0}}=1\right\} .
$$

Then, $\beta_{k} \rightarrow 0$ as $k \rightarrow \infty$.

Next, We prove Theorem 1.2. 
Proof. Let $X=X_{0}, Y=Y_{k}$ and $Z=Z_{k}$. Obvious, $I(0)=0$ and $\left(f_{4}\right)$ implies I is even. According to the previous discussion and Theorem 3.4, it suffices to show that $\left(A_{2}\right)$ and $\left(A_{3}\right)$ of Theorem 2.5 is satisfied. Since $Y$ is a finite dimensional space, all norms on $Y$ are equivalent. Thus, there exists $\gamma_{0}>0$ such that

$$
\|u\|_{p \theta} \geq \gamma\|u\|_{X_{0}}, \forall u \in Y
$$

By $\left(f_{3}\right)$, for any $M_{1}>\frac{\widetilde{\mathscr{M}}(1)}{p \gamma^{p \theta}}$, there exists $\Gamma_{0}>0$ such that

$$
F(x, t) \geq M_{1} t^{p \theta}, \forall x \in \Omega,|t| \geq \Gamma_{0}
$$

In view of $\left(f_{1}\right)$, we have

$$
|F(x, t)| \leq C\left(1+\Gamma_{0}^{q-1}\right)|t|, \forall x \in \Omega,|t| \leq \Gamma_{0},
$$

which implies

$$
F(x, t) \geq M_{1} t^{p \theta}-C^{\prime}|t|, \forall(x, t) \in \Omega \times \mathbb{R}
$$

where $C^{\prime}>0$. Therefore, from (2.1), (3.9), (4.1) and (4.2), we obtain that

$$
\begin{aligned}
I(u) & =\frac{1}{p} \widetilde{\mathscr{M}}\left(\|u\|_{X_{0}}^{p}\right)-\frac{\lambda}{p} \int_{\Omega}|u|^{p} d x-\int_{\Omega} F(x, u) d x \\
& \leq \frac{\widetilde{\mathscr{M}}(1)}{p}\|u\|_{X_{0}}^{p \theta}-\frac{\lambda}{p}\|u\|_{p}^{p}-M_{1}\|u\|_{p \theta}^{p \theta}+C^{\prime}\|u\|_{1} \\
& \leq\left(\frac{\widetilde{\mathscr{M}}(1)}{p}-\gamma^{p \theta} M_{1}\right)\|u\|_{X_{0}}^{p \theta}-\frac{\lambda}{p} C_{p}^{p}\|u\|_{X_{0}}^{p}+C^{\prime} C_{1}\|u\|_{X_{0}} .
\end{aligned}
$$

So, for any finite dimensional subspace $\widetilde{X} \subset X_{0}$, there exists $R=R(\widetilde{X}) \geq 1$ such that $I \leq 0$ as $u \in \widetilde{X} \backslash B_{\rho}$. Therefore $p \theta>p$ means the condition $\left(A_{3}\right)$ of Theorem 2.5 is satisfied.

Now, we verify the condition $\left(A_{2}\right)$ of Theorem 2.5 .

By $\left(f_{1}\right)$ and $\left(f_{5}\right)$, for any $\sigma>0$, there exists $C_{\sigma}>0$ such that

$$
F(x, t) \leq \frac{\sigma}{p}|t|^{p}+\frac{C_{\sigma}}{q}|t|^{q},
$$

for any $(x, t) \in \Omega \times \mathbb{R}$. Let

$$
\Theta=\min \left\{m_{0}, m_{0}-\frac{\lambda}{\lambda_{1}}\right\}
$$

According to Lemma 4.1 , for any fixed $\sigma>0$, we choose an integer $\widetilde{k} \geq 1$ such that

$$
\|u\|_{p}^{p} \leq \frac{2 \Theta}{3 \sigma}\|u\|_{X_{0}}^{p},\|u\|_{q}^{q} \leq \frac{q \Theta}{3 p C_{\sigma}}\|u\|_{X_{0}}^{q}
$$

for any $u \in Z_{\tilde{k}}$.

Case 1. $\frac{\lambda}{m_{0}}<\lambda_{1}$.

Choose

$$
\rho:=\|u\|_{X_{0}}=\frac{1}{3}, \quad \forall u \in Z_{\widetilde{k}} .
$$


From (4.3), (4.4) and $\left(M_{1}\right)$, we obtain

$$
\begin{aligned}
I(u) & =\frac{1}{p} \widetilde{\mathscr{M}}\left(\|u\|_{X_{0}}^{p}\right)-\frac{\lambda}{p} \int_{\Omega}|u|^{p} d x-\int_{\Omega} F(x, u) d x \\
& \geq \frac{1}{p} \widetilde{\mathscr{M}}\left(\|u\|_{X_{0}}^{p}\right)-\frac{\lambda}{p}\|u\|_{p}^{p}-\frac{C_{\sigma}}{q}\|u\|_{q}^{q}-\frac{\sigma}{p}\|u\|_{p}^{p} \\
& \left.\geq \frac{1}{p} \Theta\|u\|_{X_{0}}^{p}-\frac{1}{3 p} \Theta\right\}\|u\|_{X_{0}}^{q}-\frac{2}{3 p} \Theta\|u\|_{X_{0}}^{p}, \\
& \geq \frac{1}{3 p} \Theta\left(\|u\|_{X_{0}}^{p}-\|u\|_{X_{0}}^{q}\right) \\
& \geq \frac{1}{3 p} \Theta\left(\frac{1}{3^{p}}-\frac{1}{3^{q}}\right):=\alpha>0 .
\end{aligned}
$$

Case 2. $\frac{\lambda}{m_{0}} \geq \lambda_{1}$.

Taking into account the sequence $\lambda_{k}$ of the eigenvalues of $\mathscr{L}_{K}^{p}$ is positive and divergent, we can suppose that $\frac{\lambda}{m_{0}} \in\left[\lambda_{k-1}, \lambda_{k}\right)$ for some $k \in \mathbb{N}, k \geq 2$. We consider the variational properties of $\lambda_{k}$, denoted by

$$
\lambda_{k}=\min _{u \in Z_{k} \backslash\{0\}} \frac{\iint_{\mathbb{R}^{2 N}}|u(x)-u(y)|^{p} K(x-y) d x d y}{\int_{\Omega}|u(x)|^{p} d x} .
$$

For every $u \in Z_{\widetilde{k}} \backslash\{0\}$, we get that

$$
\begin{aligned}
I(u) & =\frac{1}{p} \widetilde{\mathscr{M}}\left(\|u\|_{X_{0}}^{p}\right)-\frac{\lambda}{p} \int_{\Omega}|u|^{p} d x-\int_{\Omega} F(x, u) d x \\
& \geq \frac{1}{p} \widetilde{\mathscr{M}}\left(\|u\|_{X_{0}}^{p}\right)-\frac{\lambda}{p}\|u\|_{p}^{p}-\frac{C_{\sigma}}{q}\|u\|_{q}^{q}-\frac{\sigma}{p}\|u\|_{p}^{p} \\
& \geq \frac{1}{p}\left(m_{0}-\frac{\lambda}{p}\right)\|u\|_{p}^{p}-\frac{C_{\sigma}}{q}\|u\|_{q}^{q}-\frac{\sigma}{p}\|u\|_{p}^{p} .
\end{aligned}
$$

Similar to Case 1, the condition $\left(A_{2}\right)$ of Theorem 2.5 is also fulfilled. The proof of Theorem 1.2 is completed.

Now we prove Theorem 1.3. According to Theorem 3.5, we only need to show the assumptions $\left(B_{1}\right)-\left(B_{3}\right)$ hold.

Step 1. Verify condition $\left(B_{1}\right)$.

In view of $p \leq p \theta<\eta \leq q<p_{s}^{*}$, we choose $\mathbb{R} \in(0,1)$ enough small such that

$$
\frac{1}{2 p} \min \left\{m_{0}, m_{0}-\frac{\lambda}{\lambda_{1}}\right\}\|u\|_{X_{0}}^{p} \geq \frac{|\beta|}{\eta} C_{\eta}^{\eta}\|u\|_{X_{0}}^{\eta}
$$

for any $u \in X_{0}$ with $\|u\|_{X_{0}} \leq \mathbb{R}$, where $C_{\eta}$ is a positive constant given in (2.1).

Next, we divide the proof into two cases.

Case I. $\frac{\lambda}{m_{0}}<\lambda_{1}$. 
We know, for $u \in Z_{k}$ with $\|u\|_{X_{0}} \leq \mathbb{R}$,

$$
\begin{aligned}
I(u) & =\frac{1}{p} \widetilde{\mathscr{M}}\left(\|u\|_{X_{0}}^{p}\right)-\frac{\lambda}{p} \int_{\Omega}|u|^{p} d x-\frac{\alpha}{\xi} \int_{\Omega}|u|^{\xi} d x-\frac{\beta}{\eta} \int_{\Omega}|u|^{\eta} d x \\
& \geq \frac{1}{p} \min \left\{m_{0}, m_{0}-\frac{\lambda}{\lambda_{1}}\right\}\|u\|_{X_{0}}^{p}-\frac{\alpha}{\xi} \beta_{k}^{\xi}\|u\|_{X_{0}}^{\xi}-\frac{|\beta|}{\eta} C_{\eta}^{\eta}\|u\|_{X_{0}}^{\eta} \\
& \geq \frac{1}{2 p} \min \left\{m_{0}, m_{0}-\frac{\lambda}{\lambda_{1}}\right\}\|u\|_{X_{0}}^{p}-\frac{\alpha}{\xi} \beta_{k}^{\xi}\|u\|_{X_{0}}^{\xi} .
\end{aligned}
$$

Choosing

$$
r_{k}:=\left(\frac{2 p \alpha \beta_{k}^{\xi}}{\xi \min \left\{m_{0}, m_{0}-\frac{\lambda}{\lambda_{1}}\right\}}\right)^{\frac{1}{p-\xi}},
$$

we have from Lemma 4.1 that $r_{k} \rightarrow 0$ as $k \rightarrow \infty$. Therefore, there exists $k_{0}>0$ such that $r_{k} \leq \mathbb{R}$ when $k \geq k_{0}$. Thus for $k \geq k_{0}$ and $u \in Z_{k}$ with $\|u\|_{X_{0}}=r_{k}$, we obtain $I(u) \geq 0$.

Case II. $\frac{\lambda}{m_{0}} \geq \lambda_{1}$.

We can follow the proof of the latter part in the verification of $\left(A_{2}\right)$. By replacing $\min \left\{m_{0}, m_{0}-\frac{\lambda}{\lambda_{1}}\right\}$ with $\left(m_{0}-\frac{\lambda}{\lambda_{k}}\right)$, and following to that of the first case, it is easy to prove that conclusion.

Step 2. Verify condition $\left(B_{2}\right)$.

For each $u \in Y_{k},\|u\|_{X_{0}}=\gamma_{k}$ with $0<\gamma_{k}<r_{k}$, we have

$$
\begin{aligned}
I(u) & =\frac{1}{p} \widetilde{\mathscr{M}}\left(\|u\|_{X_{0}}^{p}\right)-\frac{\lambda}{p} \int_{\Omega}|u|^{p} d x-\frac{\alpha}{\xi} \int_{\Omega}|u|^{\xi} d x-\frac{\beta}{\eta} \int_{\Omega}|u|^{\eta} d x \\
& \leq \frac{1}{p} \max _{0<\tau<1} \mathscr{M}(\tau)\|u\|_{X_{0}}^{p}-\frac{\lambda}{p}\|u\|_{p}^{p}-\frac{\alpha}{\xi}\|u\|_{\xi}^{\xi}-\frac{\beta}{\eta}\|u\|_{\eta}^{\eta} .
\end{aligned}
$$

As on finite dimensional spaces, all norms are equivalent. By using $\alpha>0$ and $\xi<p<\eta$, we have $I(u)<0$ for small enough $\gamma_{k}>0$.

Step 3. Verify condition $\left(B_{3}\right)$.

It follows from the verification of condition $\left(B_{1}\right)$ that, for $k \geq k_{0}$ and $u \in Z_{k}$ with $\|u\|_{X_{0}} \leq r_{k}$,

$$
I(u) \geq-\frac{\alpha}{\xi} \beta_{k}^{\xi}\|u\|_{X_{0}}^{\xi} \geq-\frac{\alpha}{\xi} \beta_{k}^{\xi} r_{k}^{\xi}
$$

Since $\beta_{k} \rightarrow 0$, and $r_{k} \rightarrow 0$ as $k \rightarrow \infty$, we have that condition $\left(B_{3}\right)$ is also satisfied. The proof of Theorem 1.3 is completed.

Now, we consider the following example as an application of the main result.

Example 4.2. Let $0<s<1<p<\infty$, ps $<N$ and $\Omega$ be an open bounded set of $\mathbb{R}^{N}$ with Lipschitz boundary. We consider the problem

$$
\begin{cases}\left(m_{0}+b\left(\iint_{\mathbb{R}^{2 N}} \frac{|u(x)-u(y)|^{p}}{|x-y|^{N+p s}} d x d y\right)^{\theta-1}\right)(-\triangle)_{p}^{s} u(x)-\lambda|u|^{p-2} u=|u|^{p \theta-1} u, & \text { in } \Omega, \\ u=0, & \text { in } \mathbb{R}^{\mathrm{N}} \backslash \Omega,\end{cases}
$$

where $m_{0}, b$ are positive constants and $\theta>1$ is also a constant.

It is clearly that

$$
\mathscr{M}(t)=m_{0}+b t^{\theta-1} \geq m_{0}>0 \text { for all } \mathrm{t} \geq 0,
$$


and

$$
\widetilde{\mathscr{M}}(t)=\int_{0}^{t} \mathscr{M}(s) d s \geq \frac{1}{\theta} \mathscr{M}(t) t \text { for all } \mathrm{t} \geq 0 .
$$

Let $f(x, t)=|t|^{p \theta-1} t$. Thus $F(x, t)=\frac{t^{p \theta+1}}{p \theta+1}$. Obviously, $f$ satisfies $\left(f_{1}\right)-\left(f_{5}\right)$. From Theorem 1.2, we obtain that the above problem has infinitely many nontrivial solutions $\left\{u_{k}\right\}$ in $X_{0}$ with unbounded energy for every $\lambda \in \mathbb{R}$.

\section{Funding}

The work was supported by the Fundamental Research Funds for Central Universities (2017B19714, 2017B07414, 2013/B19020449, 2019B44914), National Key Research and Development Program of China (2018YFC1508106), Natural Science Foundation of Jiangsu Province (BK20180500) and Scientific Research Fund of Jilin Engineering Normal University (XYB201814 and XYB201812). The work was also partially supported by Program for Innovative Research Team of Jilin Engineering Normal University, Applied mathematics research center of Jilin Engineering Normal University, the Scholarship Council and Jilin Province Science and Technology Development Plan Item (20190303117SF).

\section{REFERENCES}

[1] P. Pucci, M. Q. Xiang, B.L. Zhang, Multiple solutions for nonhomogeneous Schrödinger Kirchhoff type equations involving the fractional p-Laplacian in $\mathbb{R}^{N}$, Calc. Var. Partial Differ. Equ. 54 (2015), 2785-2806.

[2] N. Chung, Multiple solutions for a $\mathrm{p}(\mathrm{x})$-Kirchhoff-type equation with sign-changing nonlinearities, Complex Var. Elliptic Equ. 58 (2013), 1637-1646.

[3] N. Chung, Multiplicity results for a class of $\mathrm{p}(\mathrm{x})$-Kirchhoff type equations with combined nonlinearities, Electron. J. Qual. Theory Differ. Equ. 42 (2012) 1-13.

[4] J. Nie, Existence and multiplicity of nontrivial solutions for a class of Schrödinger-Kirchhofftype equations, J. Math. Anal. Appl. 417 (2014), 65-79.

[5] Y.X. Guo, J.J. Nie, Existence and multiplicity of nontrivial solutions for p-Laplacian Schrödinger- Kirchhoff-type equations, J. Math. Anal. Appl. 428 (2015), 1054-1069.

[6] M.Q. Xiang, B.L. Zhang, Degenerate Kirchhoff problems involving the fractional p-Laplacian without the (AR) condition, Complex Var. Elliptic Equ. 60 (2015), 1277-1287.

[7] M.Q. Xiang, B.L. Zhang, M. Ferrara, Existence of solutions for Kirchhoff type problem involving the non-local fractional p-Laplacian, J. Math. Anal. Appl. 424 (2015), 1021-1041.

[8] M.Q. Xiang, B.L. Zhang, V. Radulescu, Existence of solutions for perturbed fractional p-Laplacian equations, J. Differential Equations 260 (2016), 1392-413.

[9] P. Pucci, M.Q. Xiang, B.L. Zhang, Existence and multiplicity of entire solutions for fractional p-Kirchhoff equations, Adv. Nonlinear Anal. 5 (2016), 27-55.

[10] F. Colasuonno, P. Pucci, Multiplicity of solutions for p(x)-polyharmonic elliptic Kirchhoff equations, Nonlinear Anal. 74 (2011), 5962-5974.

[11] A. Fiscella, E. Valdinoci, A critical kirchhoff type problem involving a nonlocal operator, Nonlinear Anal. 94 (2014), 156-170.

[12] M. Caponi, P. Pucci, Existence theorems for entire solutions of stationary Kirchhoff fractional p-Laplacian equations, Ann. Mat. Pura Appl. 195 (2016), 2099-2129.

[13] A. Fiscella, P. Pucci, On certain nonlocal Hardy-Sobolev critical elliptic Dirichlet problems, Adv. Differential Equations 21 (2016), 571-599.

[14] A. Fiscella, P. Pucci, P-fractional Kirchhoff equations involving critical nonlinearities, Nonlinear Anal. 35 (2017), 350378.

[15] M. Ferrara, G. M. Bisci, B.L. Zhang, Existence of weak solutions for non-local fractional problems via Morse theory, Discrete Contin. Dyn. Syst. Ser. B 19 (2014), 2483-2499. 
[16] M.Q. Xiang, B.L. Zhang, X.Y. Guo, Infnitely many solutions for a fractional Kirchhoff type problem via fountain theorem, Nonlinear Anal. 120 (2015), 299-313.

[17] M.Q. Xiang, M.B. Giovanni, G.H. Tian, B.L. Zhang, Infinitely many solutions for the stationary Kirchhoff problems involving the fractional p-Laplacian, Nonlinearity. 29 (2016), 357-374.

[18] N. Nyamoradi, L.I. Zaidan, Existence of solutions for degenerate kirchhoff type problems with fractional p-Laplacian, Electron. J. Differential Equations. 2017 (2017), Article ID 115.

[19] R. Servadei, E. Valdinoci, Variational methods for non-local operators of elliptic type, Discrete Contin. Dyn. Syst. 33 (2013), 2105-2137.

[20] J. Sun, S. Liu, Nontrivial solutions of kirchhoff type problems, Appl. Math. Lett. 25 (2012), 500-504.

[21] R. Servadei, E. Valdinoci, Mountain pass solutions for non-local elliptic operators, J. Math. Anal. Appl. 389 (2012), 887-898. 